\title{
A proof of Hall's conjecture on length of ray images under starlike mappings of order $\alpha$
}

\author{
Peter Hästö and Saminathan Ponnusamy
}

\begin{abstract}
Assume that $f$ lies in the class of starlike functions of order $\alpha \in[0,1)$, that is, which are regular and univalent for $|z|<1$ and such that

$$
\operatorname{Re}\left(\frac{z f^{\prime}(z)}{f(z)}\right)>\alpha \text { for }|z|<1 .
$$
\end{abstract}

In this paper we show that for each $\alpha \in[0,1)$, the following sharp inequality holds:

$$
\left|f\left(r e^{i \theta}\right)\right|^{-1} \int_{0}^{r}\left|f^{\prime}\left(u e^{i \theta}\right)\right| d u \leq \frac{\Gamma\left(\frac{1}{2}\right) \Gamma(2-\alpha)}{\Gamma\left(\frac{3}{2}-\alpha\right)} \quad \text { for every } r<1 \text { and } \theta .
$$

This settles the conjecture of Hall (1980) positively.

\section{Todistus Hallin konjektuurille säteiden kuvien pituudesta kertaluvun $\alpha$ tähdenmuotoisissa kuvauksissa}

Tiivistelmä. Oletetaan, että $f$ on kertaluvun $\alpha \in[0,1)$ tähdenmuotoinen funktio. Tämä tarkoittaa, että $f$ on holomorfinen injektio yksikkökiekolla, jolle

$$
\operatorname{Re}\left(\frac{z f^{\prime}(z)}{f(z)}\right)>\alpha, \quad \operatorname{kun}|z|<1 .
$$

Tässä artikkelissa osoitamme, että seuraava tarkka epäyhtälö pätee jokaiselle $\alpha \in[0,1)$ :

$$
\left|f\left(r e^{i \theta}\right)\right|^{-1} \int_{0}^{r}\left|f^{\prime}\left(u e^{i \theta}\right)\right| d u \leq \frac{\Gamma\left(\frac{1}{2}\right) \Gamma(2-\alpha)}{\Gamma\left(\frac{3}{2}-\alpha\right)} \quad \text { jokaiselle } r<1 \text { ja } \theta .
$$

Tämä ratkaisee Hallin 1980 esittämän konjektuurin positiivisesti.

\section{Introduction and the main theorem}

The theory of univalent functions on domains in the complex plane $\mathbb{C}$ attracted the attention of many for more than a century, and it has been centered around the class $\mathcal{S}$ of functions $f$ regular and univalent in the unit disk $\mathbb{D}=\{z:|z|<1\}$ and normalized by the condition $f(0)=f^{\prime}(0)-1=0$. The conjecture of Bieberbach which asserted $\left|f^{(n)}(0) / n !\right| \leq n$ for all $n \geq 2$ (if $f \in \mathcal{S}$ ), was solved by de Branges [6] in 1984. The family $\mathcal{S}$ together with some of its geometric subfamilies play a key role in solving many extremal problems, and a large amount of research has been done as evidenced by the volume of articles in the literature (cf. $[10,13,14,18,24,28]$ ) and several monographs (cf. $[15,23,25]$ ). It is still an active field of research in view of several open problems and extensions in several settings [1, 19], including planar harmonic univalent mappings [9, 11].

This article concerns length of ray images under a special class of conformal mappings. Suppose that $f \in \mathcal{F} \subset \mathcal{S}$ and $f$ maps $\mathbb{D}$ onto a domain $D$. Let $C(r, \theta)$ denote the image in $D$ of the ray joining $z=0$ to $z=r e^{i \theta} \in \mathbb{D}$ under the mapping

https://doi.org/10.54330/afm.113736

2020 Mathematics Subject Classification: Primary 30C45, 30C20; Secondary 30C35.

Key words: Ray-image, length of ray-image, starlike and univalent mappings, starlike functions of order $\alpha$.

(c) 2022 The Finnish Mathematical Society 
$w=f(z)$ belonging to the family $\mathcal{F}$. Then the length $\ell(r, \theta)$ of the curve $C(r, \theta)$ is given by

$$
\ell(r, \theta):=\int_{0}^{r}\left|f^{\prime}\left(\rho e^{i \theta}\right)\right| d \rho .
$$

In 1963, Gehring and Hayman [12] showed that if $f \in \mathcal{S}^{*} \subset \mathcal{S}$, i.e. $f(\mathbb{D})$ is starlike (with respect to the origin), then there exists an absolute constant $M>0$ such that

$$
\ell(r, \theta) \leq M\left|f\left(r e^{i \theta}\right)\right| \text { for every } r<1 \text { and } \theta .
$$

We refer to this as Gehring-Hayman inequality. Motivated by this remarkable fact, Sheil-Small [29] showed that if $f \in \mathcal{S}^{*}$, then the constant $M$ in (1) can be chosen to be $1+\log 4$, and if $f \in \mathcal{S}^{*}\left(\frac{1}{2}\right) \subset \mathcal{S}^{*}:=\mathcal{S}^{*}(0)$ (see equation (2)), then the constant may be reduced to $1+\log 2$. Further investigation in this topic led Sheil-Small [29] to conjecture that if $f \in \mathcal{K} \subset \mathcal{S}^{*}\left(\frac{1}{2}\right)$, i.e. $f(\mathbb{D})$ is convex, then the correct constant is $\frac{\pi}{2}$. Hall $[16,17]$ showed that the best possible constants are 2 and $\frac{\pi}{2}$ for the families $\mathcal{S}^{*}$ and $\mathcal{S}^{*}\left(\frac{1}{2}\right)$, respectively. This settled both the conjectures of Sheil-Small. See [3] for a simpler proof of Gehring-Hayman inequality (1) with $M=2$ for the case of univalent starlike functions. At this point it is worth recalling the fact that a function belonging to $\mathcal{S}^{*}\left(\frac{1}{2}\right)$ may not be convex univalent in $|z|<R$ for any $R>\sqrt{2 \sqrt{3}-3}=0.68$. It is natural to ask for the corresponding optimal constant $M$ in (1) for several other choices of the family $\mathcal{F} \subset \mathcal{S}$.

In this article, we consider a problem posed by Hall [17]. More precisely, Hall in this paper related the following:

At the Durham Symposium on Analytic Number Theory (July 1979) Professor Hayman asked in conversation what would be the sharp bound for the class $\mathcal{S}^{*}(\alpha)$ of functions starlike of order $\alpha$, that is, which are regular and univalent for $|z|<1$ and such that

$$
\operatorname{Re}\left(\frac{z f^{\prime}(z)}{f(z)}\right)>\alpha \quad \text { for }|z|<1 .
$$

I proved in [16] that in the starlike case, that is when $\alpha=0$, this bound is 2 (sharp for the Koebe function) and it is likely that for $0<\alpha<1$ the sharp constant is

$$
\frac{\Gamma\left(\frac{1}{2}\right) \Gamma(2-\alpha)}{\Gamma\left(\frac{3}{2}-\alpha\right)} .
$$

From my result for $\alpha=0$, the upper bound $1+(1-\alpha)(\log 4)^{\alpha}$ can be derived: this is not sharp but numerically it is pretty good, for example for $\alpha=\frac{1}{2}$ it gives $1.588 \ldots$..

In view of the higher difficulty level of the problem, determining the optimal constant $M$ in (1) for other choices of the family $\mathcal{F} \subset \mathcal{S}$ is difficult and results of this type were not available for many standard geometric subclasses of the univalent family $\mathcal{S}$.

In the present paper we prove the above conjecture of Hall in full generality for the class $\mathcal{S}^{*}(\alpha)$ of functions starlike of order $\alpha, 0 \leq \alpha<1$. It is worth pointing out that the present method of proof provides also alternate proofs of the two cases, $\mathcal{S}^{*}(0)$ and $\mathcal{S}^{*}\left(\frac{1}{2}\right)$, originally settled by Hall $[16,17]$. 
Theorem 1. Suppose that $f \in \mathcal{S}^{*}(\alpha)$, i.e. $f$ is a starlike of order $\alpha$ in the unit disk $\mathbb{D}$. Then

$$
\left|f\left(r e^{i \theta}\right)\right|^{-1} \ell(r, \theta) \leq \beta(\alpha) \text { for every } r<1 \text { and } \theta,
$$

where $\ell(r, \theta):=\int_{0}^{r}\left|f^{\prime}\left(\rho e^{i \theta}\right)\right| d \rho$ and

$$
\beta(\alpha):=\frac{\Gamma\left(\frac{1}{2}\right) \Gamma(2-\alpha)}{\Gamma\left(\frac{3}{2}-\alpha\right)} .
$$

Furthermore, the constant $\beta(\alpha)$ is optimal.

We refer to $[2,20]$ for some additional research related to Hall's work and conjectures on optimal constants in the Gehring-Hayman inequality. Related to this problem, we remark that an attempt has been made by Chen and Ponnusamy [8] for sense-preserving univalent and $K$-quasiconformal harmonic mappings. In order to make a statement about what this means, we need to introduce some basic notations.

Let $f$ be a complex-valued $C^{1}$-function defined on $\mathbb{D}$ and let $\ell_{f}(\theta, r)$ be the length of the curve $\left.f\right|_{[0, z]}$, where $[0, z]$ is a radial line segment from 0 to $z=r e^{i \theta} \in \mathbb{D}$, $\theta \in[0,2 \pi]$ is fixed and $r \in[0,1)$. Then (cf. [7])

$$
\ell_{f}(\theta, r):=\ell(f([0, z]))=\int_{0}^{r}\left|d f\left(\rho e^{i \theta}\right)\right|=\int_{0}^{r}\left|f_{z}\left(\rho e^{i \theta}\right)+e^{-2 i \theta} f_{\bar{z}}\left(\rho e^{i \theta}\right)\right| d \rho .
$$

In [22], Keogh showed that if $f$ is a bounded, analytic and univalent function in $\mathbb{D}$, then, for each $\theta \in[0,2 \pi]$,

$$
\ell_{f}(\theta, r)=O(\psi(r)) \quad \text { as } r \rightarrow 1^{-},
$$

where $\psi(r)=(\log (1 /(1-r)))^{1 / 2}$ for $0<r<1$, and the exponent $1 / 2$ in $\psi(r)$ cannot be decreased. Kennedy [21] showed by examples that

$$
\ell_{f}(\theta, r)=O(\mu(r) \psi(r)) \quad \text { as } r \rightarrow 1^{-}
$$

is false in general for every positive function $\mu$ in $[0,1)$ satisfying $\mu(r) \rightarrow 0$ as $r \rightarrow 1^{-}$. In [5], Carroll and Twomey proved this result without the boundedness condition in the following form.

Theorem A. Suppose that $f(z)=a_{1} z+a_{2} z^{2}+\cdots$ is univalent in $\mathbb{D}$. Then, for any fixed $\theta \in[0,2 \pi]$, there is a constant $C_{1}>0$ such that

$$
\ell_{f}(\theta, r) \leq C_{1} \max _{\rho \in[0, r]}\left|f\left(\rho e^{i \theta}\right)\right| \psi(r) \text { for } r \in(0.5,1) .
$$

If, further, $f\left(r e^{i \theta}\right)=O(1)$ as $r \rightarrow 1^{-}$, then (5) holds.

Later, Beardon and Carne [4] gave a relatively simple argument to Theorem A in hyperbolic geometry and provided further examples. Thus, the two works of Hall mentioned in the introduction are sharper versions of this in the case of functions whose range is either a starlike domain or a convex domain. In spite of the higher level of difficulty, ideas of $[4,5]$ were considered for the class $\mathcal{S}_{H}$ of sense-preserving planar harmonic univalent mappings $f=h+\bar{g}$ in $\mathbb{D}$, with the normalization $h(0)=g(0)=0$ and $h^{\prime}(0)=1$ (see $\left.[9,10]\right)$. The family $\mathcal{S}_{H}$ together with few geometric subclasses were investigated in $[9,30]$. For further details, we refer to $[10,26]$. If the co-analytic part $g$ is identically zero in the representation $f=h+\bar{g}$, then the class $\mathcal{S}_{H}$ coincides with the family $\mathcal{S}$. Motivated by the above consideration, in 2019, Chen and Ponnusamy [8] obtained the following result for the case of planar harmonic mappings. 
Theorem B. For $K \geq 1$, let $f \in \mathcal{S}_{H}$ be a $K$-quasiconformal harmonic mapping. Then, for any fixed $\theta \in[0,2 \pi]$, there is a constant $C_{2}>0$ such that

$$
\ell_{f}(\theta, r) \leq C_{2} \max _{\rho \in[0, r]}\left|f\left(\rho e^{i \theta}\right)\right| \psi(r) \quad \text { for } r \in(0.5,1) .
$$

If, further, $f\left(r e^{i \theta}\right)=O(1)$ as $r \rightarrow 1^{-}$, then

$$
\ell_{f}(\theta, r)=O(\psi(r)) \quad \text { as } r \rightarrow 1^{-},
$$

and the exponent $1 / 2$ in $\psi(r)$ defined above cannot be replaced by a smaller number.

First we remark that Theorem B implies Theorem A when $K=1$. Secondly, the proof of Theorem B is relatively harder than the proof of Theorem A because of the fact that the arguments of Beardon and Carne [4] for Theorem A are not applicable in the proof of Theorem B.

\section{Proof of Theorem 1}

\subsection{Part 1: Proof of the main theorem.}

Lemma 1. Suppose that $f \in \mathcal{S}^{*}(\alpha)$. Then the desired inequality (3) holds whenever

$$
I(s, t)+I(t, s) \leq 2(\beta(\alpha)-1) \quad \text { for } s, t \in(0, \pi) .
$$

where

$$
\begin{aligned}
& I(s, t)=\int_{0}^{1}\left\{\frac{\sqrt{1+(1-2 \alpha)^{2} u^{2}+2(1-2 \alpha) u \cos t}}{\sqrt{1+u^{2}-2 u \cos t}}-\frac{1-(1-2 \alpha) u^{2}-2 \alpha u \cos t}{1+u^{2}-2 u \cos t}\right\} \\
& \quad \times\left\{\frac{2(1-\cos s)}{1+u^{2}-2 u \cos s}\right\}^{1-\alpha} d u .
\end{aligned}
$$

Proof. The family $\mathcal{S}^{*}(\alpha)$ is rotationally invariant in the sense that $e^{-i \theta} f\left(e^{i \theta} z\right)$ belongs to $\mathcal{S}^{*}(\alpha)$ whenever $f \in \mathcal{S}^{*}(\alpha)$. Therefore, without loss of generality, let us suppose that $\theta=0$ in (3). As a consequence, we let $h(z)=f(r z), r \in(0,1)$. Then $h$ is regular and univalent for $|z| \leq 1, h(0)=0$ and $h(1)=f(r)$. Therefore to prove (3) we have to show equivalently that

$$
\int_{0}^{1}\left|h^{\prime}(u)\right| d u \leq \beta(\alpha)|h(1)|
$$

where $\beta(\alpha)$ is defined by (4). It remains to show that (9) holds whenever (7) holds.

Now, we let $f \in \mathcal{S}^{*}(\alpha)$. Then, we have

$$
H(z):=\frac{z h^{\prime}(z)}{h(z)}=\frac{r z f^{\prime}(r z)}{f(r z)} \quad \text { and } \quad \operatorname{Re} H(z)>\alpha, \quad z=r e^{i \theta} \in \overline{\mathbb{D}} .
$$

Using the Herglotz representation theorem for regular functions with positive real part (cf. $[14,24,28]$ ) and the fact that $h \in \mathcal{S}^{*}(\alpha)$, we also have, for $z \in \mathbb{D}$,

$$
H(z)=\frac{z h^{\prime}(z)}{h(z)}=\frac{1}{2 \pi} \int_{-\pi}^{\pi} \frac{1+(1-2 \alpha) z e^{-i t}}{1-z e^{-i t}} d V(t)
$$

where $V(t)$ is an increasing function for $t \in[-\pi, \pi]$ which satisfies $\frac{V(\pi)-V(-\pi)}{2 \pi}=1$. Therefore, using standard arguments and some computations, we find that

$$
H(u)=\int_{0}^{\pi} \frac{1+(1-2 \alpha) u e^{-i t}}{1-u e^{-i t}} d W(t)
$$


and

$$
\frac{\partial}{\partial u} \log |h(u)|=u^{-1} \operatorname{Re} H(u)=\int_{0}^{\pi} \frac{1-(1-2 \alpha) u^{2}-2 \alpha u \cos t}{u\left(1+u^{2}-2 u \cos t\right)} d W(t),
$$

where $W(t):=\frac{V(t)-V(-t)}{2 \pi}$. Note that $W(0)=0, W(\pi)=1$ and $W$ is increasing on $[0, \pi]$ and so $d W$ is nonnegative and has a total mass 1 . Using (10) it follows that

$$
\begin{aligned}
|H(u)|-\operatorname{Re} H(u) \leq & \int_{0}^{\pi}\left[\frac{\sqrt{1+(1-2 \alpha)^{2} u^{2}+2(1-2 \alpha) u \cos t}}{\sqrt{1+u^{2}-2 u \cos t}}\right. \\
& \left.-\frac{1-(1-2 \alpha) u^{2}-2 \alpha u \cos t}{1+u^{2}-2 u \cos t}\right] d W(t) .
\end{aligned}
$$

Next we note from the definition of $H(z)$ that

$$
\begin{aligned}
\int_{0}^{1}\left|h^{\prime}(u)\right| d u & =\int_{0}^{1}|H(u)||h(u)| u^{-1} d u \\
& =\int_{0}^{1} \operatorname{Re} H(u)|h(u)| u^{-1} d u+\int_{0}^{1}[|H(u)|-\operatorname{Re} H(u)]|h(u)| u^{-1} d u .
\end{aligned}
$$

Regarding the first integral on the right, we find by (11) that

$$
\int_{0}^{1} \operatorname{Re} H(u)|h(u)| u^{-1} d u=\int_{0}^{1}|h(u)| \frac{\partial}{\partial u} \log |h(u)| d u=|h(1)| .
$$

We then estimate the second of the integrals in (13). From (11) we also have

$$
\begin{aligned}
\log \left\{\frac{|h(u)|}{|h(1)|}\right\} & =\int_{1}^{u} \frac{\partial}{\partial v} \log |h(v)| d v=\int_{1}^{u} v^{-1} \operatorname{Re} H(v) d v \\
& =\int_{0}^{\pi} \int_{1}^{u} \frac{1-(1-2 \alpha) v^{2}-2 \alpha v \cos t}{v\left(1+v^{2}-2 v \cos t\right)} d v d W(t) \\
& =\int_{0}^{\pi} \log \left\{\frac{u(2-2 \cos t)^{1-\alpha}}{\left(1+u^{2}-2 u \cos t\right)^{1-\alpha}}\right\} d W(t)
\end{aligned}
$$

Applying Jensen's inequality [31, p. 24] and performing exponentiation on both sides of the last relation, we get

$$
|h(u)| u^{-1} \leq|h(1)| \int_{0}^{\pi}\left\{\frac{2(1-\cos t)}{1+u^{2}-2 u \cos t}\right\}^{1-\alpha} d W(t) .
$$

Therefore, from (12) and (15) we deduce that

$$
\begin{aligned}
\int_{0}^{1}\{|H(u)|-\operatorname{Re} H(u)\}|h(u)| u^{-1} d u & \leq|h(1)| \int_{0}^{\pi} \int_{0}^{\pi} I(s, t) d W(t) d W(s) \\
& \leq \frac{|h(1)|}{2} \int_{0}^{\pi} \int_{0}^{\pi}[I(s, t)+I(t, s)] d W(t) d W(s),
\end{aligned}
$$

where $I(s, t)$ is given by $(8)$.

Thus to complete the proof of the inequality (9), using (13), (14) and (16), it suffices to show

$$
\sup \{I(s, t)+I(t, s): 0 \leq t \leq \pi, 0 \leq s \leq \pi\} \leq 2(\beta(\alpha)-1) .
$$

2.2. Part 2: Proof of the inequality (7). To establish the inequality (7), we need to evaluate the integrals $I(t, s)$ and $I(s, t)$, where $I(t, s)$ is defined by $(8)$. In 
order to do this, we rewrite (8) in the following form

$$
I(s, t)=[2(1-\cos s)]^{1-\alpha}[J(t, s)-K(t, s)],
$$

where

$$
J(s, t)=\int_{0}^{1} \frac{\sqrt{(1+(1-2 \alpha) u)^{2}-2(1-2 \alpha) u(1-\cos t)}}{\sqrt{\left(1+u^{2}-2 u \cos t\right)}\left(1+u^{2}-2 u \cos s\right)^{1-\alpha}} d u
$$

and

$$
K(s, t)=\int_{0}^{1} \frac{\left[1-(1-2 \alpha) u^{2}-2 \alpha u \cos t\right]}{\left(1+u^{2}-2 u \cos t\right)\left(1+u^{2}-2 u \cos s\right)^{1-\alpha}} d u .
$$

In order to prove the inequality (7), we need to establish several lemmas.

Let us denote $S:=2(1-\cos s), T:=2(1-\cos t)$ and $\gamma:=1-2 \alpha$ so that $S, T \in(0,4)$ and $\gamma \in(-1,1]$. Then (18) can be written in terms of $S$ and $T$, which we denote by $I(S, T)$ for obvious reason, and thus, we have

$I(S, T)=\int_{0}^{1}\left(\frac{S}{(1-u)^{2}+S u}\right)^{\frac{1+\gamma}{2}}\left[\frac{\sqrt{(1+\gamma u)^{2}-\gamma T u}}{\sqrt{(1-u)^{2}+T u}}-\frac{1-\gamma u^{2}-(1-\gamma)\left(1-\frac{T}{2}\right) u}{(1-u)^{2}+T u}\right] d u$.

Our first aim is to give an upper bound for the sum $I(S, T)+I(T, S)$ in terms of a simpler integrand. We begin by giving the bound for the first term in the square bracket factor in the integrand of $I(S, T)$.

Lemma 2. For $T \in(0,4), \gamma \in(-1,1]$ and $u \in(0,1)$,

$$
\frac{\sqrt{(1+\gamma u)^{2}-\gamma T u}}{\sqrt{(1-u)^{2}+T u}} \leq \frac{1+\gamma}{2} \frac{1+u}{\sqrt{(1-u)^{2}+T u}}+\frac{1-\gamma}{2} .
$$

Proof. The claim is clear if $\gamma=1$, so we assume that $\gamma \in(-1,1)$. As

$$
1+\gamma u=\frac{1+\gamma}{2}(1+u)+\frac{1-\gamma}{2}(1-u)
$$

we calculate

$$
\frac{1+\gamma u}{\sqrt{(1-u)^{2}+T u}}=\frac{1+\gamma}{2} \frac{1+u}{\sqrt{(1-u)^{2}+T u}}+\frac{1-\gamma}{2} \frac{1-u}{\sqrt{(1-u)^{2}+T u}} .
$$

Subtracting this from the inequality in the statement of the lemma, we see that the claim (19) is equivalent to

$$
\frac{\sqrt{(1+\gamma u)^{2}-\gamma T u}-(1+\gamma u)}{\sqrt{(1-u)^{2}+T u}} \leq \frac{1-\gamma}{2}\left[1-\frac{1-u}{\sqrt{(1-u)^{2}+T u}}\right],
$$

or, multiplied by $\frac{1}{1-\gamma} \sqrt{(1-u)^{2}+T u}$,

$$
\frac{1}{1-\gamma}\left[\sqrt{(1+\gamma u)^{2}-\gamma T u}-(1+\gamma u)\right] \leq \frac{1}{2}\left[\sqrt{(1-u)^{2}+T u}-(1-u)\right] .
$$

When $\gamma \geq 0$, the left-hand side of (20) is non-positive, so the claim is clear, and therefore, we may assume that $\gamma<0$ and denote $b:=-\gamma>0$, where $0<b<1$. When $T=0$, both sides equal 0 , so the inequality holds. We may next rewrite $(20)$ equivalently as $\varphi(T) \geq 0$, where

$$
\varphi(T)=\frac{1}{2}\left[\sqrt{(1-u)^{2}+T u}-(1-u)\right]-\frac{1}{1+b}\left[\sqrt{(1-b u)^{2}+b T u}-(1-b u)\right] .
$$


We observed that $\varphi(0)=0$ and thus it suffices to show that $\varphi$ is increasing on $(0,4)$. We calculate

$$
\varphi^{\prime}(T)=\frac{1}{4} \frac{u}{\sqrt{(1-u)^{2}+T u}}-\frac{1}{2(1+b)} \frac{b u}{\sqrt{(1-b u)^{2}+b T u}}
$$

and it is non-negative when

$$
\sqrt{(1-b u)^{2}+b T u} \geq \frac{2 b}{1+b} \sqrt{(1-u)^{2}+T u} .
$$

Because $1+b \geq 2 \sqrt{b}$, the last inequality holds if

$$
\sqrt{(1-b u)^{2}+b T u} \geq \sqrt{b} \sqrt{(1-u)^{2}+T u} .
$$

Squaring both sides gives the equivalent condition

$$
(1-b u)^{2}+b T u \geq b\left((1-u)^{2}+T u\right) \Longleftrightarrow 1-b \geq b(1-b) u^{2},
$$

which holds since $b \in(0,1)$ and $u \in(0,1)$. Thus, $\varphi(T) \geq \varphi(0)=0$ and the proof of the lemma is complete.

Lemma 3. Let $a:=\frac{T}{S}, a \in(0, \infty)$. Then with $S, T \in(0,4)$ and $I(S, T)$ defined as above, we have

$$
I(S, T)+I(T, S) \leq \frac{1+\gamma}{2} \int_{0}^{\infty}\left[(a+w)^{-\frac{1+\gamma}{2}}+\left(\frac{1}{a}+w\right)^{-\frac{1+\gamma}{2}}\right] \frac{\sqrt{1+w}-1}{1+w} w^{-\frac{1-\gamma}{2}} d w
$$

where $\gamma \in(-1,1]$.

Proof. By Lemma 2, we recall that

$$
\frac{\sqrt{(1+\gamma u)^{2}-\gamma T u}}{\sqrt{(1-u)^{2}+T u}} \leq \frac{1+\gamma}{2}\left(\frac{1+u}{\sqrt{(1-u)^{2}+T u}}\right)+\frac{1-\gamma}{2} .
$$

For the numerator of the integrand of $K(s, t)$ with the change of variables as in the beginning of Subsection 2, i.e. precisely the second term of the square-bracked term in the expression of $I(S, T)$, we use

$$
1-\gamma u^{2}-(1-\gamma)\left(1-\frac{T}{2}\right) u=\frac{1+\gamma}{2}\left(1-u^{2}\right)+\frac{1-\gamma}{2}\left((1-u)^{2}+T u\right)
$$

so that

$$
\frac{1-\gamma u^{2}-(1-\gamma)\left(1-\frac{T}{2}\right) u}{(1-u)^{2}+T u}=\frac{1+\gamma}{2}\left(\frac{1-u^{2}}{(1-u)^{2}+T u}\right)+\frac{1-\gamma}{2} .
$$

Using these relations, we can therefore estimate

$$
\begin{aligned}
& \frac{\sqrt{(1+\gamma u)^{2}-\gamma T u}}{\sqrt{(1-u)^{2}+T u}}-\frac{1-\gamma u^{2}-(1-\gamma)\left(1-\frac{T}{2}\right) u}{(1-u)^{2}+T u} \\
& \leq \frac{1+\gamma}{2}\left[\frac{1+u}{\sqrt{(1-u)^{2}+T u}}-\frac{1-u^{2}}{(1-u)^{2}+T u}\right] \\
& =\frac{1+\gamma}{2}\left[\frac{1}{\sqrt{(1-u)^{2}+T u}}-\frac{1-u}{(1-u)^{2}+T u}\right](1+u) .
\end{aligned}
$$


Thus, we have established the inequality

$$
\begin{aligned}
I(S, T) & \leq \frac{1+\gamma}{2} \int_{0}^{1}\left(\frac{S}{(1-u)^{2}+S u}\right)^{\frac{1+\gamma}{2}}\left[\frac{1}{\sqrt{(1-u)^{2}+T u}}-\frac{1-u}{(1-u)^{2}+T u}\right](1+u) d u \\
& =\frac{1+\gamma}{2} \int_{0}^{1}\left(\frac{S}{1+\frac{S u}{(1-u)^{2}}}\right)^{\frac{1+\gamma}{2}}\left[\frac{1}{\sqrt{1+\frac{T u}{(1-u)^{2}}}}-\frac{1}{1+\frac{T u}{(1-u)^{2}}}\right] \frac{1+u}{(1-u)^{2+\gamma}} d u
\end{aligned}
$$

and for $I(T, S)$, it follows similarly that

$$
I(T, S) \leq \frac{1+\gamma}{2} \int_{0}^{1}\left(\frac{T}{1+\frac{T u}{(1-u)^{2}}}\right)^{\frac{1+\gamma}{2}}\left[\frac{1}{\sqrt{1+\frac{S u}{(1-u)^{2}}}}-\frac{1}{1+\frac{S u}{(1-u)^{2}}}\right] \frac{1+u}{(1-u)^{2+\gamma}} d u .
$$

Let us continue with the change of variables

$$
w:=\frac{T u}{(1-u)^{2}} .
$$

Then

so that

$$
d w=T \frac{1+u}{(1-u)^{3}} d u
$$

$$
\begin{aligned}
I(S, T) & \leq \frac{1+\gamma}{2} \int_{0}^{1}\left(\frac{S}{1+\frac{S}{T} w}\right)^{\frac{1+\gamma}{2}}\left[\frac{1}{\sqrt{1+w}}-\frac{1}{1+w}\right] \frac{1+u}{(1-u)^{2+\gamma}} d u \\
& =\frac{1+\gamma}{2} \int_{0}^{\infty}\left(\frac{\frac{S}{T}}{1+\frac{S}{T} w}\right)^{\frac{1+\gamma}{2}} \frac{\sqrt{1+w}-1}{1+w}\left[T^{-\frac{1}{2}}(1-u)\right]^{1-\gamma} d w .
\end{aligned}
$$

From the relation $(1-u)^{2}=\frac{T}{w} u$, or equivalently the quadratic equation

$$
w(1-u)^{2}+T(1-u)-T=0,
$$

we solve for $1-u$ with the restriction $0<u<1$ :

$$
1-u=\frac{1}{2}\left(-\frac{T}{w}+\sqrt{\left(\frac{T}{w}\right)^{2}+4 \frac{T}{w}}\right)=\frac{\sqrt{T^{2}+4 T w}-T}{2 w}=\frac{2 T}{\sqrt{T^{2}+4 T w}+T}
$$

so that

$$
1-u=\frac{2 \sqrt{T}}{\sqrt{T+4 w}+\sqrt{T}} \leq \frac{\sqrt{T}}{\sqrt{w}}, \quad \text { i.e., } \quad T^{-\frac{1}{2}}(1-u) \leq \frac{1}{\sqrt{w}} .
$$

Therefore, we conclude that

$$
I(S, T) \leq \frac{1+\gamma}{2} \int_{0}^{\infty}\left(\frac{1}{a+w}\right)^{\frac{1+\gamma}{2}} \frac{\sqrt{1+w}-1}{1+w} w^{-\frac{1-\gamma}{2}} d w
$$

where $a=\frac{T}{S} \in(0, \infty)$. Interchanging the role of $S$ and $T$ in the above proof gives an analogous inequality for $I(T, S)$ :

$$
I(T, S) \leq \frac{1+\gamma}{2} \int_{0}^{\infty}\left(\frac{1}{b+w}\right)^{\frac{1+\gamma}{2}} \frac{\sqrt{1+w}-1}{1+w} w^{-\frac{1-\gamma}{2}} d w, \quad b=\frac{S}{T} \in(0, \infty) .
$$

Note that $b=1 / a$. Finally, adding these two estimates, we obtain the desired claim. 
Let us next consider the expression in the case $\gamma=1$. Based on previous research, it is already known that the expression in Lemma 3 is maximized when $a=1$. However, we will need the monotonicity, which is a stronger claim.

Lemma 4. The function

$$
a \longmapsto \int_{0}^{\infty}\left[\frac{1}{a+w}+\frac{1}{\frac{1}{a}+w}\right] \frac{\sqrt{1+w}-1}{1+w} d w=: G_{1}(a)
$$

is increasing in $(0,1)$.

Proof. It turns out that we can explicitly calculate the integrals involved in the expression. Since

$$
\frac{1}{(a+w)(1+w)}=\frac{1}{1-a}\left[\frac{1}{a+w}-\frac{1}{1+w}\right]
$$

we calculate

$$
\int_{0}^{\infty} \frac{d w}{(a+w)(1+w)}=\left.\frac{1}{1-a} \ln \left(\frac{a+w}{1+w}\right)\right|_{0} ^{\infty}=\frac{\ln \frac{1}{a}}{1-a}
$$

Similarly,

$$
\int_{0}^{\infty} \frac{d w}{\left(\frac{1}{a}+w\right)(1+w)}=\frac{\ln a}{1-\frac{1}{a}}=\frac{a \ln \frac{1}{a}}{1-a} .
$$

The other two integrals are more complicated, but we find that

$$
\int \frac{d w}{\left(\frac{1}{a}+w\right) \sqrt{1+w}}=2 \sqrt{\frac{a}{1-a}} \tan ^{-1}\left(\sqrt{\frac{a}{1-a}} \sqrt{1+w}\right)+C
$$

When we use the formula for the term with $a+w$, the number inside the arctangent is imaginary, so we use also the formula

$$
\tan ^{-1}(z)=\frac{i}{2} \log \left(\frac{i+z}{i-z}\right)
$$

Hence we conclude

$$
\int \frac{d w}{(a+w) \sqrt{1+w}}=-\sqrt{\frac{1}{1-a}} \log \left(\frac{\sqrt{1-a}+\sqrt{1+w}}{\sqrt{1-a}-\sqrt{1+w}}\right)+C .
$$

With these integral functions, we obtain that

$$
\begin{aligned}
& \int_{0}^{\infty}\left[\frac{1}{a+w}+\frac{1}{\frac{1}{a}+w}\right] \frac{\sqrt{1+w}-1}{1+w} d w=-\frac{1+a}{1-a} \ln \frac{1}{a} \\
& +2 \sqrt{\frac{a}{1-a}}\left[\frac{\pi}{2}-\tan ^{-1}\left(\sqrt{\frac{a}{1-a}}\right)\right]-\sqrt{\frac{1}{1-a}}\left[\log (-1)-\log \left(\frac{\sqrt{1-a}+1}{\sqrt{1-a}-1}\right)\right] \\
& =2 \sqrt{\frac{a}{1-a}} \tan ^{-1}\left(\sqrt{\frac{1-a}{a}}\right)+\sqrt{\frac{1}{1-a}} \log \left(\frac{1+\sqrt{1-a}}{1-\sqrt{1-a}}\right)-\frac{1+a}{1-a} \ln \frac{1}{a} .
\end{aligned}
$$

The graph of this function, i.e. $G_{1}(a)$, is shown in Figure 1.

We need to show that this expression is increasing in $a$. We change variables by defining $b:=\sqrt{\frac{1-a}{a}}$ so that $a=\frac{1}{1+b^{2}}$ and our expression equals

$$
G(b):=\frac{2}{b} \tan ^{-1}(b)+2 \frac{\sqrt{1+b^{2}}}{b} \log \left(b+\sqrt{1+b^{2}}\right)-\frac{2+b^{2}}{b^{2}} \ln \left(1+b^{2}\right), \quad b \in(0, \infty) .
$$




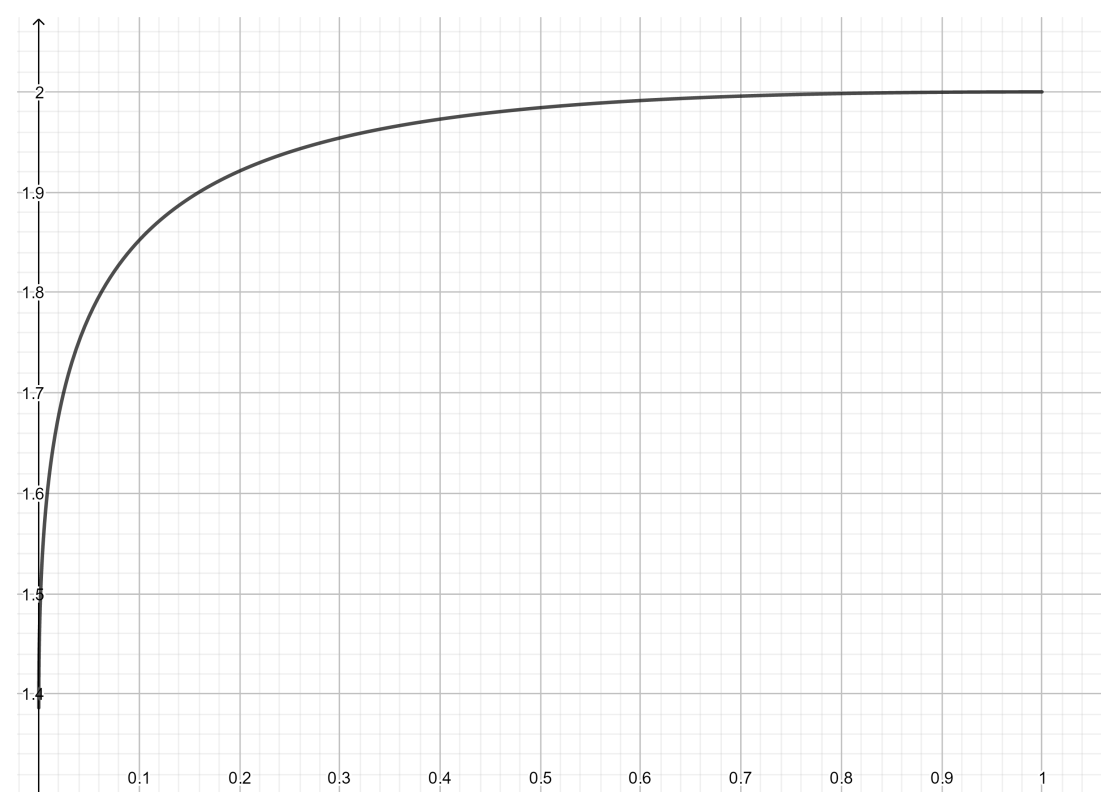

Figure 1. The graph of the function $G_{1}(a)$ from Lemma 4.

Since $b$ is decreasing in $a$, we establish our claim by showing that $G$ is decreasing on $(0, \infty)$. We calculate

$$
\begin{aligned}
\frac{1}{2} G^{\prime}(b) & =-\frac{1}{b^{2}} \tan ^{-1}(b)+\frac{1}{b\left(1+b^{2}\right)}-\frac{\log \left(b+\sqrt{1+b^{2}}\right)}{b^{2} \sqrt{1+b^{2}}}+\frac{1}{b}+\frac{2}{b^{3}} \ln \left(1+b^{2}\right)-\frac{2+b^{2}}{b\left(1+b^{2}\right)} \\
& =-\frac{1}{b^{2}} \tan ^{-1}(b)-\frac{\log \left(b+\sqrt{1+b^{2}}\right)}{b^{2} \sqrt{1+b^{2}}}+\frac{2}{b^{3}} \ln \left(1+b^{2}\right) .
\end{aligned}
$$

Hence it suffices to show that

$$
g(b):=\frac{b^{2}}{2} G^{\prime}(b)=-\tan ^{-1}(b)-\frac{\log \left(b+\sqrt{1+b^{2}}\right)}{\sqrt{1+b^{2}}}+\frac{2}{b} \ln \left(1+b^{2}\right)
$$

is negative. We see that $g\left(0^{+}\right)=0$, and show that $g$ is decreasing on $(0, \infty)$. A calculation gives

$$
\begin{aligned}
g^{\prime}(b) & =-\frac{1}{1+b^{2}}+b \frac{\log \left(b+\sqrt{1+b^{2}}\right)}{\left(1+b^{2}\right)^{\frac{3}{2}}}-\frac{1}{1+b^{2}}-\frac{2}{b^{2}} \ln \left(1+b^{2}\right)+\frac{4}{1+b^{2}} \\
& =\frac{2}{1+b^{2}}+b \frac{\log \left(b+\sqrt{1+b^{2}}\right)}{\left(1+b^{2}\right)^{\frac{3}{2}}}-\frac{2}{b^{2}} \ln \left(1+b^{2}\right) .
\end{aligned}
$$

With the new variable $c:=b^{2}$, we find that

$$
h(c):=c g^{\prime}(\sqrt{c})=\frac{2 c}{1+c}+\left(\frac{c}{1+c}\right)^{\frac{3}{2}} \log (\sqrt{c}+\sqrt{1+c})-2 \ln (1+c), \quad c \in(0, \infty) .
$$

We need to show that $h$ is negative on $(0, \infty)$, and we observe that $h\left(0^{+}\right)=0$. To show that $h$ is decreasing on $(0, \infty)$, we calculate the derivative

$$
\begin{aligned}
h^{\prime}(c) & =\frac{2}{(1+c)^{2}}+\frac{3}{2}\left(\frac{c}{1+c}\right)^{\frac{1}{2}} \frac{\log (\sqrt{c}+\sqrt{1+c})}{(1+c)^{2}}+\frac{c}{2(1+c)^{2}}-\frac{2}{1+c} \\
& =-\frac{3}{2} \frac{c}{(1+c)^{2}}+\frac{3}{2}\left(\frac{c}{1+c}\right)^{\frac{1}{2}} \frac{\log (\sqrt{c}+\sqrt{1+c})}{(1+c)^{2}}
\end{aligned}
$$


from which we define a function $k$ by

$$
k(c):=\frac{2}{3}\left(1+c^{2}\right)^{\frac{5}{2}} c^{-\frac{1}{2}} h^{\prime}(c)=-\sqrt{c(1+c)}+\log (\sqrt{c}+\sqrt{1+c}), \quad c \in(0, \infty) .
$$

Finally, we observe that $k\left(0^{+}\right)=0$ and

$$
k^{\prime}(c)=-\frac{1+2 c}{2 \sqrt{c(1+c)}}+\frac{1}{2 \sqrt{c(1+c)}}=-\sqrt{\frac{c}{1+c}} \leq 0 \text { : for } c \in(0, \infty) .
$$

Thus $k(c)<0$ for $c \in(0, \infty)$, so that $h$ is decreasing on $(0, \infty)$ and thus negative, which implies that $g$ is decreasing and negative for $c \in(0, \infty)$. Hence, $G$ is decreasing on $(0, \infty)$, which is equivalent to the original claim. This completes the proof of the lemma.

We are now ready to continue our investigation on Lemma 3, again.

Lemma 5. The maximum of the right-hand side in Lemma 3 is achieved when $a=1$, so that

$$
I(S, T)+I(T, S) \leq(1+\gamma) \int_{0}^{\infty}\left[(1+w)^{-\frac{2+\gamma}{2}}-(1+w)^{-\frac{3+\gamma}{2}}\right] w^{-\frac{1-\gamma}{2}} d w
$$

where $\gamma \in(-1,1]$.

Proof. We consider the function

$$
G(a):=\int_{0}^{\infty}\left[(a+w)^{-\frac{1+\gamma}{2}}+\left(\frac{1}{a}+w\right)^{-\frac{1+\gamma}{2}}\right] \frac{\sqrt{1+w}-1}{1+w} w^{-\frac{1-\gamma}{2}} d w
$$

where $\gamma \in(-1,1]$ and $a \in(0, \infty)$. We need to show that $G$ is maximized by $a=1$. To that end, we consider the derivative with respect to $a$ :

$$
\begin{aligned}
\frac{2}{1+\gamma} G^{\prime}(a) & =\int_{0}^{\infty}\left[-(a+w)^{-\frac{3+\gamma}{2}}+a^{-2}\left(\frac{1}{a}+w\right)^{-\frac{3+\gamma}{2}}\right] \frac{\sqrt{1+w}-1}{1+w} w^{-\frac{1-\gamma}{2}} d w \\
& =-\int_{0}^{\infty}\left(\frac{w}{a+w}\right)^{\frac{3+\gamma}{2}} \frac{\sqrt{1+w}-1}{(1+w) w^{2}} d w+\int_{0}^{\infty} \frac{1}{a^{2}}\left(\frac{w}{\frac{1}{a}+w}\right)^{\frac{3+\gamma}{2}} \frac{\sqrt{1+w}-1}{(1+w) w^{2}} d w .
\end{aligned}
$$

In the first integral we use the change of variables $v:=\frac{w}{a}$ and this gives

$$
\int_{0}^{\infty}\left(\frac{w}{a+w}\right)^{\frac{3+\gamma}{2}} \frac{\sqrt{1+w}-1}{(1+w) w^{2}} d w=\frac{1}{a} \int_{0}^{\infty}\left(\frac{v}{1+v}\right)^{\frac{3+\gamma}{2}} \frac{\sqrt{1+a v}-1}{(1+a v) v^{2}} d v,
$$

whereas in the second one we use $v:=a w$ and obtain

$$
\int_{0}^{\infty} \frac{1}{a^{2}}\left(\frac{w}{\frac{1}{a}+w}\right)^{\frac{3+\gamma}{2}} \frac{\sqrt{1+w}-1}{(1+w) w^{2}} d w=\frac{1}{a} \int_{0}^{\infty}\left(\frac{v}{1+v}\right)^{\frac{3+\gamma}{2}} \frac{\sqrt{1+\frac{v}{a}}-1}{\left(1+\frac{v}{a}\right) v^{2}} d v .
$$

Therefore, we have the following expression for the derivative

$$
\frac{2 a}{1+\gamma} G^{\prime}(a)=\int_{0}^{\infty}\left(\frac{v}{1+v}\right)^{\frac{3+\gamma}{2}} \frac{1}{v^{2}}\left[\frac{\sqrt{1+\frac{v}{a}}-1}{1+\frac{v}{a}}-\frac{\sqrt{1+a v}-1}{1+a v}\right] d v .
$$

Denote

$$
g(x):=x^{-\frac{1}{2}}-x^{-1}
$$

and observe that the square bracket term equals $g\left(1+\frac{v}{a}\right)-g(1+v a)$. We find that

$$
g^{\prime}(x)=-\frac{1}{2} x^{-\frac{3}{2}}+x^{-2}=\frac{1}{2}(2-\sqrt{x}) x^{-2}
$$


so that $g$ is increasing on $[0, \sqrt{2}]$ and decreasing on $[\sqrt{2}, \infty)$. When $a<1$, we have $1+\frac{v}{a}>1+a v$ and so it follows that $v \mapsto g\left(1+\frac{v}{a}\right)-g(1+v a)$ is positive until some value $v_{0}$ and then negative. Furthermore, the function

$$
v \mapsto\left(\frac{v}{1+v}\right)^{-\frac{1-\gamma}{2}}
$$

is decreasing on $(0, \infty)$. Therefore, we have

$$
\left[g\left(1+\frac{v}{a}\right)-g(1+v a)\right]\left(\frac{v}{1+v}\right)^{-\frac{1-\gamma}{2}} \geq\left[g\left(1+\frac{v}{a}\right)-g(1+v a)\right]\left(\frac{v_{0}}{1+v_{0}}\right)^{-\frac{1-\gamma}{2}}
$$

both when $v \leq v_{0}$ and $v \geq v_{0}$. We conclude that

$$
\frac{2 a}{1+\gamma} G^{\prime}(a) \geq\left(\frac{v_{0}}{1+v_{0}}\right)^{-\frac{1-\gamma}{2}} \int_{0}^{\infty}\left(\frac{v}{1+v}\right)^{2} \frac{1}{v^{2}}\left[\frac{\sqrt{1+\frac{v}{a}}-1}{1+\frac{v}{a}}-\frac{\sqrt{1+a v}-1}{1+a v}\right] d v
$$

Up to a constant, the right-hand side of (23) is the derivative of the function in the case $\gamma=1$. By Lemma 4 , this function is increasing on $(0,1)$, so its derivative, and hence the right-hand side of the inequality in (23) above, is non-negative. It follows that $G^{\prime}(a) \geq 0$ on $(0,1)$. Furthermore, by symmetry we conclude that $G^{\prime}(a) \leq 0$ on $(1, \infty)$. Hence the maximum of $G$ occurs at $a=1$, as claimed.

Finally, we are ready to prove that the inequality (7) holds when $\alpha \in[0,1)$.

2.3. Proof of Theorem 1. To prove (17) it suffice to show that

$$
\sup \{I(S, T)+I(T, S): 0 \leq S \leq 4,0 \leq T \leq 4\} \leq 2(\beta(\alpha)-1) .
$$

Recall that $\gamma=1-2 \alpha$ and so, by Lemma 5, to suffices to show equivalently that

$$
(1-\alpha) \int_{0}^{\infty}\left[(1+w)^{\alpha-\frac{3}{2}}-(1+w)^{\alpha-2}\right] w^{-\alpha} d w \leq \frac{\Gamma\left(\frac{1}{2}\right) \Gamma(2-\alpha)}{\Gamma\left(\frac{3}{2}-\alpha\right)}-1
$$

by the definition of $\beta(\alpha)$. We then consider the beta function (not the function $\beta(\alpha)$ from before), and its relation to the gamma function as follows

$$
\int_{0}^{\infty} t^{x-1}(1+t)^{-x-y} d t=\frac{\Gamma(x) \Gamma(y)}{\Gamma(x+y)} .
$$

We use this formula with $x=1-\alpha$ and $y=\frac{1}{2}$ or $y=1$. This gives that

$$
\begin{aligned}
& (1-\alpha) \int_{0}^{\infty}\left[(1+w)^{\alpha-\frac{3}{2}}-(1+w)^{\alpha-2}\right] w^{-\alpha} d w \\
& =(1-\alpha)\left[\frac{\Gamma\left(\frac{1}{2}\right) \Gamma(1-\alpha)}{\Gamma\left(\frac{3}{2}-\alpha\right)}-\frac{\Gamma(1) \Gamma(1-\alpha)}{\Gamma(2-\alpha)}\right]=\frac{\Gamma\left(\frac{1}{2}\right) \Gamma(2-\alpha)}{\Gamma\left(\frac{3}{2}-\alpha\right)}-1,
\end{aligned}
$$

since $(1-\alpha) \Gamma(1-\alpha)=\Gamma(2-\alpha)$ and $\Gamma(1)=1$. This completes the proof of the desired estimate (7), which, by Lemma 1 implies that the Gehring-Hayman inequality holds with constant $\beta(\alpha)$.

It remains to be shown that $\beta(\alpha)$ given by (4) cannot be replaced by any smaller constant. We show that the extremal function for our problem is $k_{\alpha}$ defined by $k_{\alpha}(z):=z /(1-z)^{2-2 \alpha}$. We calculate that

$$
k_{\alpha}^{\prime}(z)=\frac{1+(1-2 \alpha) z}{(1-z)^{3-2 \alpha}} \quad \text { and } \quad \frac{z k_{\alpha}^{\prime}(z)}{k_{\alpha}(z)}=\alpha+(1-\alpha) \frac{1+z}{1-z} .
$$


From this we see that $k_{\alpha} \in \mathcal{S}^{*}(\alpha)$. As before, we set $\gamma=1-2 \alpha$ and we have that

$$
\left|k_{\alpha}^{\prime}\left(r e^{i \theta}\right)\right|=\frac{\sqrt{1+(\gamma r)^{2}+2 \gamma r \cos \theta}}{\left(1+r^{2}-2 r \cos \theta\right)^{1+\frac{\gamma}{2}}} .
$$

Furthermore, $\left|k_{\alpha}\left(e^{i \theta}\right)\right|=(2(1-\cos \theta))^{-\frac{1+\gamma}{2}}$. Let us denote again $T:=2(1-\cos \theta)$. Then we have shown that

$$
\lim _{r \rightarrow 1} \frac{\ell(r, \theta)}{\left|k_{\alpha}\left(r e^{i \theta}\right)\right|}=T^{\frac{1+\gamma}{2}} \int_{0}^{1} \frac{\sqrt{(1+\gamma u)^{2}-\gamma T u}}{\left((1-u)^{2}+T u\right)^{1+\frac{\gamma}{2}}} d u .
$$

We are interested in the limit value of the right-hand side when $T \rightarrow 0$. For some small $\epsilon>0$, we restrict the integral to the range $u \in(1-\epsilon, 1)$ for a lower bound, and estimate

$$
\sqrt{(1+\gamma u)^{2}-\gamma T u} \geq(1+\gamma)(1-O(\epsilon+T)) .
$$

We estimate the remaining terms with the same change of variables $w:=\frac{T u}{(1-u)^{2}}$ as before:

$$
\int_{1-\epsilon}^{1} \frac{T^{\frac{1+\gamma}{2}}}{\left((1-u)^{2}+T u\right)^{1+\frac{\gamma}{2}}} d u \geq \int_{T(1-\epsilon) / \epsilon^{2}}^{\infty} \frac{T^{\frac{-1+\gamma}{2}}(1-u)^{1-\gamma}}{(2-\epsilon)(1+w)^{1+\frac{\gamma}{2}}} d w .
$$

Also as before, solving for $T^{-\frac{1}{2}}(1-u)$ and using $T \leq \epsilon w$ (which follows from $u \geq 1-\epsilon$ ), we have:

$$
T^{-\frac{1}{2}}(1-u)=\frac{2}{\sqrt{T+4 w}+\sqrt{T}} \geq \frac{1}{\sqrt{\epsilon / 4+1}+\sqrt{\epsilon / 4}} \frac{1}{\sqrt{w}}=(1-O(\sqrt{\epsilon})) \frac{1}{\sqrt{w}} .
$$

With the previous two estimates, we obtain

$$
\lim _{T \rightarrow 0} \int_{1-\epsilon}^{1} \frac{T^{\frac{1+\gamma}{2}} \sqrt{(1+\gamma u)^{2}-\gamma T u}}{\left((1-u)^{2}+T u\right)^{1+\frac{\gamma}{2}}} d u \geq(1-O(\sqrt{\epsilon})) \frac{1+\gamma}{2} \int_{0}^{\infty} w^{-\frac{1-\gamma}{2}}(1+w)^{-1-\frac{\gamma}{2}} d w
$$

so that

$$
\begin{aligned}
\lim _{\theta \rightarrow 0} \lim _{r \rightarrow 1} \frac{\ell(r, \theta)}{\left|k_{\alpha}\left(r e^{i \theta}\right)\right|} & \geq(1-O(\sqrt{\epsilon}))(1-\alpha) \int_{0}^{\infty} w^{-\alpha}(1+w)^{\alpha-\frac{3}{2}} d w \\
& =(1-O(\sqrt{\epsilon})) \frac{\Gamma\left(\frac{1}{2}\right) \Gamma(2-\alpha)}{\Gamma\left(\frac{3}{2}-\alpha\right)} .
\end{aligned}
$$

The claim follows from this as $\epsilon \rightarrow 0$.

Acknowledgements. This work was completed during the visit of the second author to the University of Turku, Finland in 2019-2020, and this author thanks Prof. Matti Vuorinen for his continuous support and his encouragement. The visit of this author was supported by a grant under "India-Finland Joint Call for Mobility of Researchers Programme" and this author acknowledges the support received from the Department of Science \& Technology, Government of India, and the Academy of Finland.

Compliance with ethical standards. The authors declare that they do not have conflict of interests. The research complies with ethical standards. 


\section{References}

[1] Avkhadiev, F. G., and K.-J. Wirths: Schwarz-Pick type inequalities. - Frontiers Math., Birkhäuser Verlag, Basel, 2009.

[2] Balasubramanian, R., V. Karunakaran, and S. Ponnusamy: A proof of Hall's conjecture on starlike mappings. - J. London Math. Soc. 48:2, 1993, 271-282.

[3] Balasubramanian, R., and S. Ponnusamy: An alternate proof of Hall's theorem on a conformal mappings inequality. - Bull. Belgium Math. Soc. 3, 1996, 209-213.

[4] Beardon, A. F., and T. K. Carne: Euclidean and hyperbolic lengths of images of arcs. Proc. London Math. Soc. 97, 2008, 183-208.

[5] Carroll, T., and J. B. Twomey: Conformal mappings of close-to-convex domains. - J. London Math. Soc. 55, 1997, 489-498.

[6] DE Branges, L.: A proof of the Bieberbach conjecture. - Acta Math. 154, 1985, 137-152.

[7] Chen, S. L., G. Liu, and S. Ponnusamy: Linear measure and $K$-quasiconformal harmonic mappings. - Sci. Sin. Math. 47, 2017, 1-10 (in Chinese).

[8] Chen, S.L., and S. Ponnusamy: Radial length, radial John disks and $K$-quasiconformal harmonic mappings. - Potential Anal. 50, 2019, 415-437.

[9] Clunie, J. G., and T. Sheil-Small: Harmonic univalent functions. - Ann. Acad. Sci. Fenn. Ser. A I 9, 1984, 3-25.

[10] Duren, P. L.: Univalent functions. - Springer-Verlag, New York, 1983.

[11] Duren, P.: Harmonic mappings in the plane. - Cambridge Univ. Press, New York, 2004.

[12] Gehring, F. W., and W. K. Hayman: An inequality in the theory of conformal mapping. J. Math. Pures Appl. 41, 1963, 353-361.

[13] Goluzin, G. M.: Geometric theory of functions of a complex variable. - Transl. Math. Monogr. 26, Amer. Math. Soc. Providence, 1969.

[14] Goodman, A. W.: Univalent functions. Volumes I and II. - Mariner, Tampa, FL, 1983.

[15] Graham, I., and G. Kohr: Geometric function theory in one and higher dimensions. - Marcel Dekker Inc., New York, 2003.

[16] Hall, R. R.: The length of ray-images under starlike mappings. - Mathematika 23, 1976, 147-150.

[17] HaLl, R. R.: A conformal mapping inequality for starlike functions of order $\frac{1}{2}$. - Bull. London Math. Soc. 12, 1980, 119-126.

[18] Hayman, W. K.: Multivalent functions. Second edition. - Cambridge Tracts in Math. 110, Cambridge Univ. Press, Cambridge, 1994.

[19] Hayman, W. K., and E. F. Lingham: Research problems in function theory. Fiftieth anniversary edition. - Problem Books in Mathematics, Springer, Cham, 2019.

[20] Karunakaran, V.: Length of ray-images under conformal maps. - Proc. Amer. Math. Soc. 87, 1983, 289-294.

[21] Kennedy, P. B.: Conformal mapping of bounded domains. - J. London Math. Soc. 31, 1956, $332-336$.

[22] KeOgh, F.R.: A property of bounded schlicht functions. - J. London Math. Soc. 29, 1954, $379-382$.

[23] Lehto, O.: Univalent functions and Teichmüller spaces. - Grad. Texts in Math. 109, SpringerVerlag, NewYork, 1987.

[24] Pommerenke, Ch.: Univalent functions. With a chapter on quadratic differentials by Gerd Jensen. - Studia Mathematica/Mathematische Lehrbücher 15, Vandenhoeck and Ruprecht, Göttingen, 1975.

[25] Pommerenke, Ch.: Boundary behaviour of conformal maps. - Springer, New York, 1992. 
[26] Ponnusamy, S., and A. Rasila: Planar harmonic and quasiregular mappings. - In: Topics in Modern Function Theory (eds. St. Ruscheweyh and S. Ponnusamy), Chapter in CMFT, RMS-Lecture Notes Series No. 19, 2013, 267-333.

[27] Robertson, M. I. S.: On the theory of univalent functions. - Ann. of Math. (2) 37:2, 1936, 374-408.

[28] Ruscheweyh, St.: Convolutions in geometric function theory. - Les Presses de l'Université de Montréal, Montréal, 1982.

[29] Sheil-Small, T.: Some conformal mapping inequalities for starlike and convex functions. - J. London Math. Soc. 1:2, 1969, 577-587.

[30] Sheil-Small, T.: Constants for planar harmonic mappings. - J. London Math. Soc. 42, 1990, $237-248$.

[31] Zygmund, A.: Trigonometric series. - Cambridge Univ. Press, London and New York, 1968.

Received 23 March 2021 • Accepted 27 May 2021 • Published online 24 January 2022

Peter Hästö

University of Turku

Department of Mathematics and Statistics FI-20014 University of Turku, Turku, Finland peter.hasto@utu.fi
Saminathan Ponnusamy

Indian Institute of Technology Madras

Department of Mathematics

Chennai-600 036, India

samy@iitm.ac.in 